\title{
Serum chromium, glycosylated hemoglobin level and body mass index among control and uncontrol sudanese patients with type II diabetes mellitus: Correlation study
}

\author{
Nassr Eldin M. A. shrif 1, Ahmed Mohamed Ahmed Dawood 1, ${ }^{*}$, Abdelmahmoud Mokhtar Bashir 2, Hassan \\ Mohammed Khair Ibrahim ${ }^{3}$ and Abd Elgadir A Altoum ${ }^{4}$ \\ ${ }^{1}$ Clinical chemistry - Al-Zaiem Al-Azhari University, Sudan. \\ ${ }^{2}$ Department of Hematology, Faculty of Medical Laboratory Sciences, Sudan University of Science and Technology, \\ Khartoum, Sudan. \\ ${ }^{3}$ Medical Laboratory Sciences, college of health sciences, Gulf Medical University, AL JURF - Ajman - United Arab Emirates.
}

GSC Biological and Pharmaceutical Sciences, 2021, 16(02), 196-203

Publication history: Received on 30 June 2021; revised on 22 August 2021; accepted on 24 August 2021

Article DOI: https://doi.org/10.30574/gscbps.2021.16.2.0219

\begin{abstract}
Background: Chromium is an essential micronutrient which is required for the normal functioning of insulin and regulation of blood sugar levels. It acts as a vital antioxidant for maintaining insulin homeostasis. In diabetes mellitus, the free radical production is increased and levels of antioxidants like chromium, vanadium, selenium and manganese are reduced.
\end{abstract}

Aim: To study the level of serum chromium level in patients with type 2 diabetes mellitus and its association with glycemic control.

Materials and Methods: One hundred and twenty individuals were enrolled in this study, classified into 60 type II diabetes mellitus (type II DM) patients and 60 apparently health as control group. Serum chromium and Glycosylated Hemoglobin (HbA1c) level were measured using atomic absorption spectrometry and Ichroma. Results: The results showed significant decrease in serum chromium level in type II DM patients $(0.0151 \pm 0.005)$ when compared with healthy group $(0.122 \pm 0.691)$ with $p$-value $(0.002)$. In addition to that there was significant decrease in mean concentration of serum chromium level in controlled diabetic patients $(0.0206 \pm 0.003)$ when compared with uncontrolled diabetic patients $(0.0120 \pm 0.002)$ with P-value $(0.04)$.Also there was significant positive correlation between chromium level and Body Mass Index (BMI) (R-value 0.450, P-value 0.014), and significant negative correlation between chromium level and age (R-value- 0.660, P-value 0.011), a significant strong negative correlation between chromium level and HbA1c (R-value -0.843, P-value 0.0260).

Conclusion: The study concluded that, serum chromium level is significantly decrease in type II DM.

Keywords: Type II diabetes; Chromium; Glycosylated Hemoglobin; Body Mass Index

\section{Introduction}

Chromium, an essential trace element as well as a vital antioxidant, plays an important role in glucose and lipid metabolism by improving glucose intolerance and lowering elevated lipids. Chromium deficiency affects the maintenance of normal glucose tolerance and healthy lipid profiles [1]. Chromium deficient type 2 diabetic were noted to have decreased insulin binding \& reduced number of insulin receptors, thereby leading to glucose intolerance [2].

\footnotetext{
${ }^{*}$ Corresponding author: Abd Elgadir A Altoum

Medical Laboratory Sciences, college of health sciences, Gulf Medical University, AL JURF - Ajman - United Arab Emirates.
} 
Diabetes mellitus is a group of metabolic diseases in which there are high blood sugar levels over a prolonged period. Symptoms of high blood sugar include frequent urination, increased thirst, and increased hunger. If left untreated, diabetes can cause many complications. Acute complications include diabetic ketoacidosis and nonketotic hyperosmolar coma. Serious long-term complications include cardiovascular disease, stroke, kidney failure, foot ulcers and damage to the eyes [3]. Chromium deficiency also manifests separately as neuropathy, encephalopathy and impaired immune response. As age advances, the levels of chromium in hair, sweat and blood decreases significantly [4].

Diabetes mellitus is the most common disease in Sudan, is having an increasing impact on rates of morbidity and mortality in Sudan. Because the highest prevalence of diabetes in our Sudanese communities and the ignorance of its victims, this research was carried out to help in management of diabetes to prevent complication .Chromium an essential trace element and has important role in insulin action .chromium deficiency cause impaired in insulin action which lead to develop diabetes mellitus. Therefore, chromium uses as supplement for treated DM, lower blood lipid levels and promote weight loss. Accordingly, the present study conducted to estimate serum chromium level among Sudanese patients with type $2 \mathrm{DM}$ and its correlation with study variables.

\section{General objective}

- To evaluate serum chromium level in Sudanese diabetic patients with type II DM.

- Specific objective:

- To estimate serum chromium level among Sudanese patients with type 2 diabetes mellitus.

- To compare between serum chromium level in diabetic patients and non-diabetic.

- To compare between serum chromium level in controlled diabetic patients and uncontrolled diabetic patients.

- To correlate between serum chromium level and study variable (age, sex, BMI, HbA1c and Duration of the disease).

\section{Material and methods}

\subsection{Study design}

This is a descriptive analytical cross-sectional study.

\subsection{Study area}

The study was conducted in Khartoum state in Jaber abo alaez hospital.

\subsection{Study period}

This study was conducted from February to May 2021.

\subsection{Study population}

The study subjects consist of 120 persons, classified in to 60 healthy apparently as control group and 60 patients with type $2 \mathrm{DM}$, then they divided in to 22 controlled diabetic group (HbA1c $\leq 7.0 \%$ ) and 38 uncontrolled diabetic group $(\mathrm{HbA} 1 \mathrm{c}>7.0 \%)$.

\subsection{Inclusion criteria}

Sudanese patients with type 2 DM.

\subsection{Exclusion criteria}

Patients with renal failure, hepatic failure, cardiac failure, thyroid disorders, alcoholics, lactating and pregnant females and those who were under treatment with chromium supplements.

\subsection{Sampling}

$5(\mathrm{ml})$ of blood was collected by standard procedure, then it divided in to $2.5(\mathrm{ml})$ placed in plain containers and $2.5(\mathrm{ml})$ placed in Ethylenediaminetetraacetic acid (EDTA) containers. 


\subsection{Ethical consideration}

- permission was obtained from concerned authorities before conducting the study

- the objective was explained to all individual participating in this study

\subsection{Data analysis}

Data collected was analyzed by using the SPSS computer program ,the mean of chromium level in different group were comparison, t-test was used to compare between study variable and Bivariate Pearson's correlation was used to determine the correlation between variables.

\section{Methods}

\subsection{Estimation of chromium}

Chromium was estimated by atomic absorption spectrometry.

\subsection{Principle of method for chromium}

The technique makes use of absorption spectrometry to assess the concentration of analyte in a sample it requires standards with known analyte content to establish the relation between the measured absorbance and the analyte concentration and relies therefore on the beer-lambert law. In short, the electrons of the atoms in the atomizer can be promoted to higher orbital (excited state) for short period of time by absorbing a defined quantity of energy (radiation of given wavelength).this amount of energy, wavelength, is specific to a particular electron element. In general each wavelength corresponds to only one element, .the radiation flux without a sample and with a sample in the atomizer is measured using detector, and the ratio between the two values (the absorbance) is converted to analyst concentration or mass using the Beer Lambert Law.

\subsection{Estimation of HbA1c}

HbA1c was estimated by Ichroma.

\subsection{Principle}

Ichroma $\mathrm{HbA1c}$ is based on florescence immunoassay technology, specifically the sandwich immune-detection method.

Whole blood is added to the mixture of hemolysis buffer and detection buffer, which result in hemolysis of red blood cells. Such that by mixing detecting buffer with blood specimen in test tube, the florescence-labeled detector anti- HbA1c antibody in buffer binds to HbA1c antigen in blood specimen. The sample mixture is loaded and migrates on the matrix of test cartilage; the complexes of detector antibody and HbA1c are captured to anti-HbA1c sandwich pair antibody that has been immobilized on test matrix. As a result, the higher concentration of HbA1c produces a higher florescence signal from HbA1c-antibody complexes. The signal is interpreted and the result display on Ichroma Reader in units of $\%(N G S P)$.

\subsection{Procedure}

- From hemolysis buffer $(100 \mu \mathrm{l})$ was added into detection buffer tube.

- Whole blood $(5 \mu \mathrm{l})$ was added to detection buffer tube then shacked 15 times.

- From sample mixture $(75 \mu \mathrm{l})$ was taken and dispensed into sample well on the test cartridge.

- $\quad$ Test cartridge inserted into I chamber for 12 minutes then inserted into Ichroma ${ }^{\mathrm{TM}}$ Reader for scanning.

- The button (select) was pressed to start scanning process and the test result read on display screen.

\subsection{Quality control}

The precision and accuracy of the methods used in this study were cheeked each time a batch was analyzed by including commercial prepared control sera.

\subsection{Calculation of body mass index}

BMI obtained by calculation according to formula:

$$
\text { Weight (kg) } \div \text { height (2) (m) }
$$




\section{Results}

In this study 120 subjects were chosen for estimation of serum chromium and HbA1c, they classified as 60 healthy apparently as controlled group and 60 patients with type II DM, then they divided in to controlled diabetic group (HbA1c $\leq 7.0 \%$ )and uncontrolled diabetic group (HbA1c $>7.0 \%$ ) The results showed significant decrease in serum chromium level in type $2 \mathrm{DM}$ patients $(0.0151 \pm 0.005)$ when compared with healthy group $(0.122 \pm 0.691)$ with p-value 0.002 (Table $3)$. In addition There was significant decrease in mean concentration of serum chromium level in controlled diabetic patients $(0.0206 \pm 0.003)$ when compared with uncontrolled diabetic patients $(0.0120 \pm 0.002)$ with P-value $(0.04)$ (Table 3) Also there was significant positive correlation between chromium level and BMI (R-value 0.450, P-value 0.014) Figure (3), and significant negative correlation between chromium level and age (R-value- 0.660, P-value 0.011) Figure (2) .while there is no correlation between chromium level and HbA1c (R-value -0.843, P-value 0.0260) Figure (5).

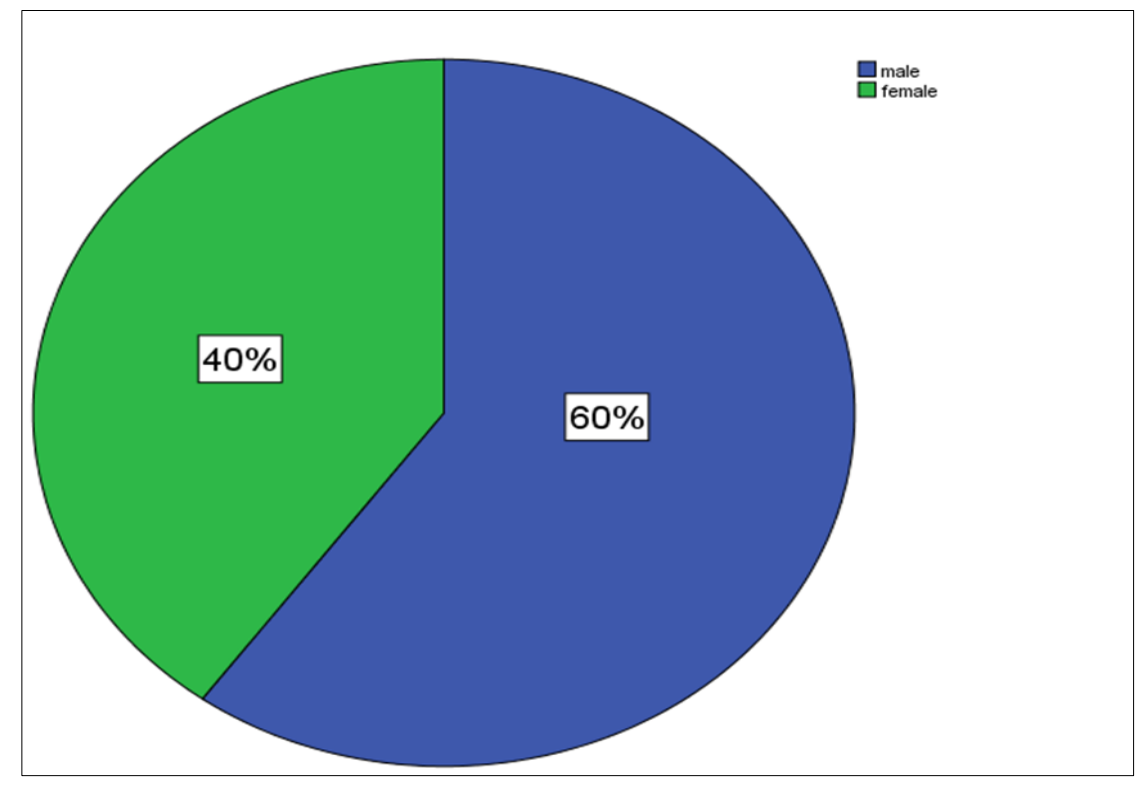

Figure 1 Frequencies of gender among diabetic patients, result expressed as percentage (\%)

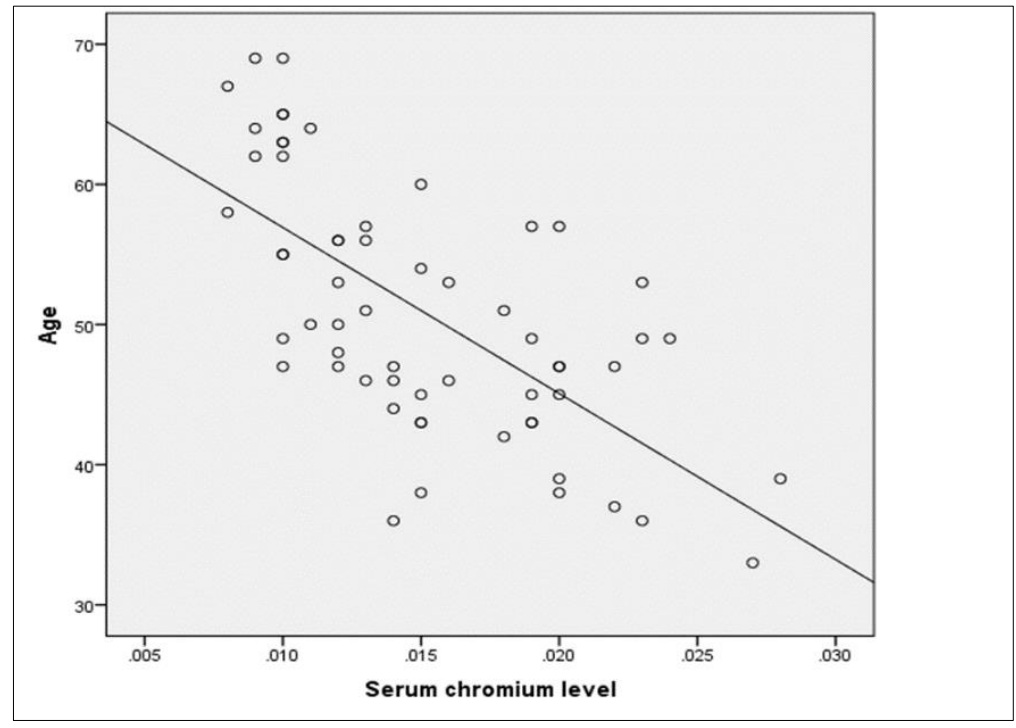

Figure 2 Correlation between serum chromium level and age of diabetic patients $(r=-0.660, P$-value $=0.011)$ 
GSC Biological and Pharmaceutical Sciences, 2021, 16(02), 196-203

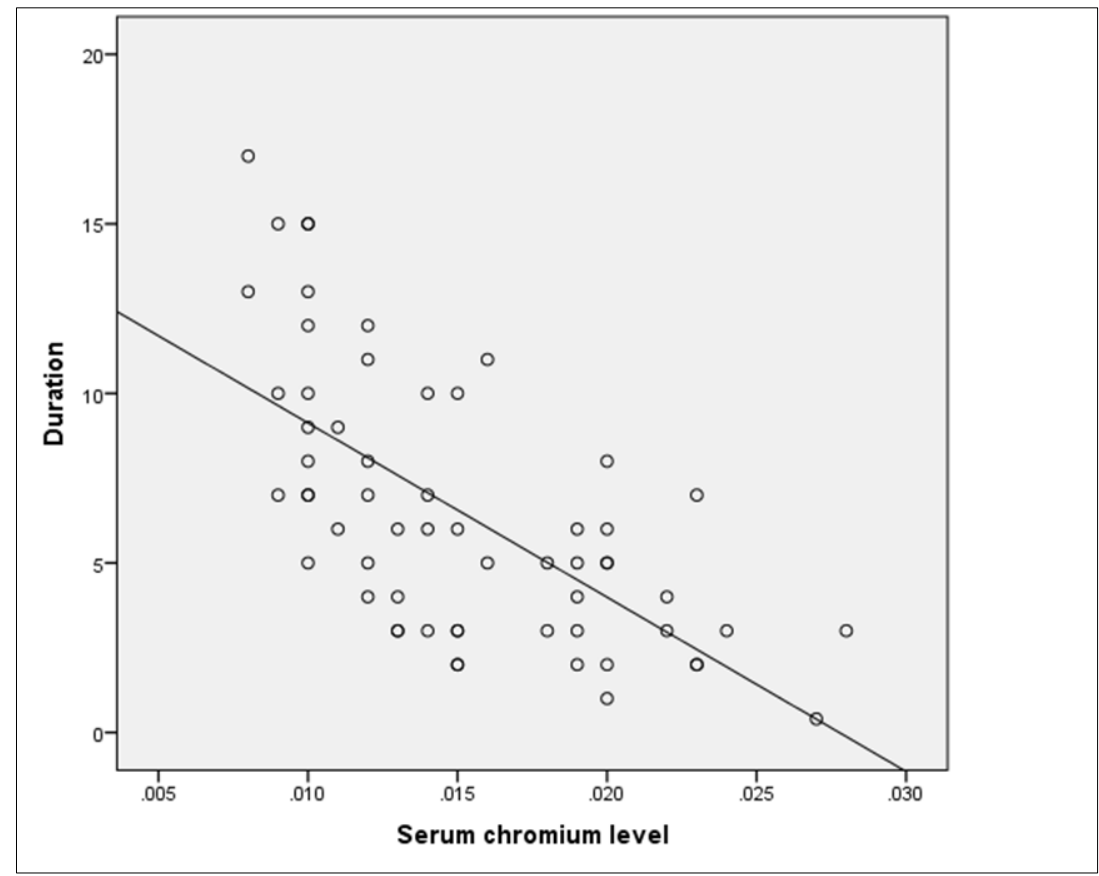

Figure 3 Correlation between serum chromium level and duration of diabetes mellitus $(r=-0.649, \mathrm{P}-\mathrm{value}=0.022)$

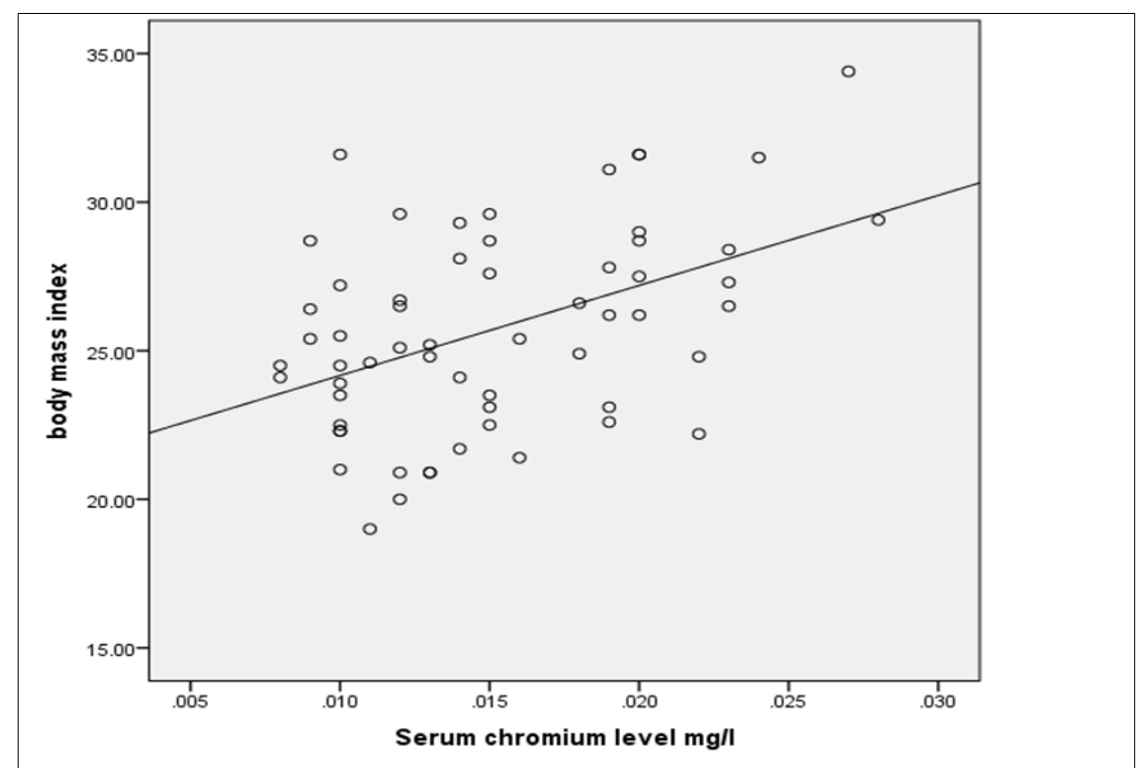

Figure 4 Correlation between serum chromium level and BMI in diabetic patients $(r=0.450, \mathrm{P}$-value $=0.014)$

Table 1 Mean concentration of serum chromium level in case and control groups

\begin{tabular}{|c|c|c|c|}
\hline \multirow{2}{*}{ Variable } & Mean \pm SD & P-value \\
\hline \multirow{2}{*}{ Chromium } & Case $(\mathrm{n}=60)$ & $0.0151 \pm 0.005$ & \multirow{2}{*}{0.002} \\
\cline { 2 - 3 } & Control $(\mathrm{n}=60)$ & $0.122 \pm 0.691$ & \\
\hline
\end{tabular}


Table 2 Mean concentration of serum chromium level in controlled diabetic patients and uncontrolled diabetic patients

\begin{tabular}{|c|c|c|c|}
\hline $\begin{array}{c}\text { Serum } \\
\text { Chromium } \\
\text { concentration }\end{array}$ & $\begin{array}{c}\text { Controlled Diabetic } \\
\text { patients } \\
(\mathbf{n}=\mathbf{2 2})\end{array}$ & $\begin{array}{c}\text { Uncontrolled } \\
\text { Diabetic patients } \\
\mathbf{( n = 3 8 )}\end{array}$ & P-value \\
\hline Mean \pm SD & $0.0206 \pm 0.003$ & $0.0120 \pm 0.002$ & 0.04 \\
\hline
\end{tabular}

$\mathrm{t}$-test was used to calculate P value; P value less than 0.05 considered significant; Mean \pm Std. Deviation

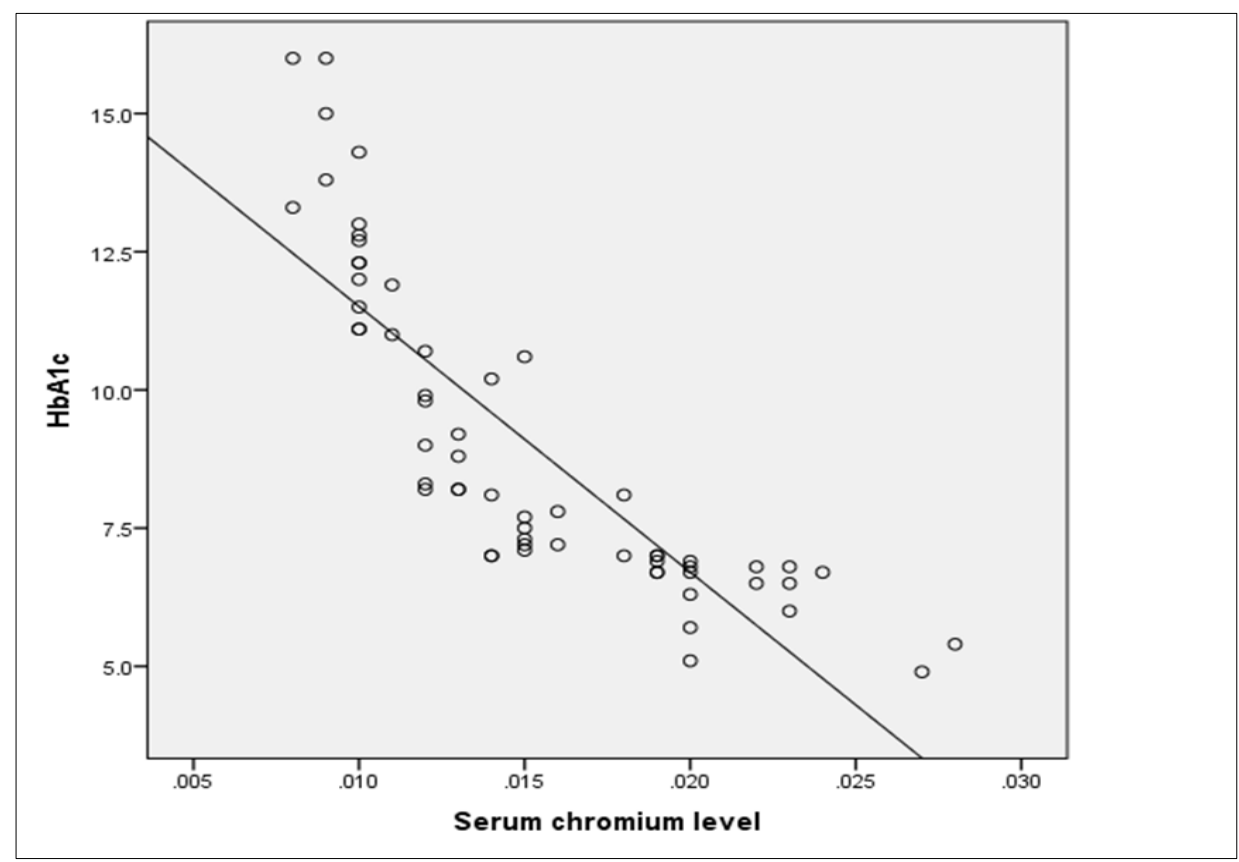

Figure 5 Correlation between serum chromium level and HbA1c in diabetic patients $(r=-0.843, \mathrm{P}-\mathrm{value}=0.0260)$

Table 3 Mean of chromium, HbA1c, BMI and age among gender variation in diabetic patients

\begin{tabular}{|l|c|c|c|}
\hline \multicolumn{2}{|l|}{ Study variable } & $\begin{array}{c}\text { Mean } \\
\text { Deviation }\end{array}$ & \multirow{2}{*}{ P value } \\
\hline \multirow{2}{*}{ Chromium } & Male & $0.0145 \pm 0.005$ & \multirow{2}{*}{0.193} \\
\cline { 2 - 3 } & Female & $0.0162 \pm 0.004$ & \\
\hline \multirow{2}{*}{ HbA1c } & Male & $9.22 \pm 2.7$ & \multirow{2}{*}{0.517} \\
\cline { 2 - 3 } & Female & $8.72 \pm 3.1$ & \multirow{2}{*}{0.684} \\
\hline \multirow{2}{*}{ BMI } & Male & $25.5 \pm 3.6$ & \\
\cline { 2 - 3 } & Female & $25.9 \pm 3$ & \\
\hline Age & & $50.8 \pm 9$ & \\
\hline
\end{tabular}

t-test was used to calculate P value; P value less than 0.05 considered significant; Mean \pm Std. Deviation 


\section{Discussion}

Diabetes mellitus is one of the most important causes of death all over the world because of its adverse effects on cardiovascular system and is one of the important factors that may influence metabolic status.

The present study showed significant decrease in mean serum chromium level in diabetic patients with type 2 when compared with control subject, the findings agreed with previous study which reported that, Lower plasma chromium level was associated with hyperglycemia, hyperinsulinemia, hypertension, insulin resistance and c.reactive protein (CRP). High plasma Cr was also associated with favorable fat distribution: WHtR, WC and WHR were significantly lower at high plasma Cr levels. Plasma chromium concentration therefore ameliorate glucose and lipid metabolism. High plasma chromium there reduces cardiovascular risk [5]. The mechanisms by which Cr metabolism affects plasma insulin and CRP levels are unclear, even though some studies have shown it improves insulin sensitivity Kelley-Hedgepeth etal $[6,7]$. A lower mean of serum chromium level was noticed among good controlled diabetic when compared to uncontrolled diabetic, which was however not statistically significant.

The study showed no significant difference in mean serum chromium level in males when compared to females, This is in line with studies by Rajendran K, Manikandan S et al in their study the mean serum chromium levels were lower in females when compared to males which was however insignificant $(\mathrm{p}=0.3487)$ which showed no significant difference in chromium levels between males and females, The findings confirmed by the present study showed that, there insignificant difference in mean concentration of chromium in type 2 DM females when compared with males group [8]Also the result study showed no significant difference in mean concentration of HbA1c in males compare to female. These findings agreed with previous study conducted in America which found insignificant impact with HbA1c value in both male and female [9].In addition the present study results show there was a significant correlation between serum chromium level and BMI, these findings agreed with previous study conducted with significant effect on either body weight or BMI. Strong association between serum chromium and superoxide dismutase (SOD) in relation to HbA1c in this study gives a strong point that these variables could be used as markers of cell injury with the intention in further part of life route to progressive complications in type II DM [10]. Also, there was no correlation between chromium level and duration of type II DM.

In our study we observed a strong negative correlation between serum chromium level and HbA1c in diabetic patients this in agree with a study conducted in Sudan that reported by Abd Elgadir A. Altoum, and Mohamed, H. Eltom, et al $[11,12]$ concluded that significant correlation between serum Chromium level with fasting blood sugar, Glycosylated Hemoglobin (HbA1c), Total Cholesterol and Triglycerides among type 2 diabetes patients. Deficient chromium level, as their vital role in lipids metabolism and insulin function chromium supplementation are essential in the control of glycemic metabolism and lipid profile in DM also agree with new study conducted in Bangladesh which reported On correlation analysis, serum Cr level showed significant negative correlation with FSG and $\mathrm{HbA}_{1} \mathrm{C}$ levels in type 2 diabetic patients. The study reflected that serum chromium level was reduced in DM which was higher with glycemic status of this disease. Categorization of patients with type $2 \mathrm{DM}$ on the basis of low serum Cr level and high FSG and $\mathrm{HbA}_{1} \mathrm{c}$ level will be helpful for risk of diabetic complications [13].

\section{Conclusion}

Serum chromium levels were reduced in type II diabetes mellitus patients irrespective of their HbA1c value. The study indicated that they are significant decrease in chromium level among Sudanese patients with type II DM. Deficient chromium level, as their vital role in lipids metabolism and insulin function chromium supplementation are essential in the control of glycemic metabolism in DM.

\section{Compliance with ethical standards}

\section{Disclosure of conflict of interest}

There was no conflict of interest in this study. Statement of informed consent Informed consent was obtained from all individual participants included in the study.

\section{Statement of informed consent}

Informed consent was obtained from all individual participants included in the study. 


\section{References}

[1] Lewicki S, Zdanowski R, Krzyzowska M, Lewicka A, Debski B, Niemcewicz M, et al. The role of Chromium III in the organism and its possible use in diabetes and obesity treatment. Ann AgriEnv Med. 2014; 2: 331-35.

[2] Cefalu WT, Hu FB. Role of Chromium in Human Health and in Diabetes.Diabetes Care. 2004; 27(11): $2741-51$.

[3] Kitabchi AE, Umpierrez GE, Miles JM, Fisher JN. Hyperglycemic crises in adult patients with diabetes. Diabetes Care. 2009; 32(7): 1335-43.

[4] Davis S, McLaren Howard J, Hunnisett A, Howard M. Age related decrease in Chromium levels in 51,665 hair, sweat and serum samples from 40782 patients-implications for prevention of cardiovascular disease and type 2 diabetes. Metabolism. 2019; 46(5): 469-73.

[5] Ngala RA, Awe MA, Nsiah P. The effects of plasma chromium on lipid profile, glucose metabolism and cardiovascular risk in type 2 diabetes mellitus. A case - control study. PLoS One. 5 Jul 2018; 13(7): e0197977.

[6] Kelley-Hedgepeth A, Lloyd-Jones DM, Colvin A, Matthews KA, Johnston J, Sowers MFR, et al. Ethnic differences in C-reactive protein concentrations. ClinChem. 2008; 54:1027-1037.

[7] Sahin K, Onderci M, Tuzcu M, Ustundag B, Cikim G, Ozercan IH, et al. Effect of chromium on carbohydrate and lipid metabolism in a rat model of type 2 diabetes mellitus: the fat-fed, streptozotocin-treated rat Metabolism. 2007; 56: 1233-1240.

[8] Rajendran K, Manikandan S, Nair LD, Karuthodiyil R, Vijayarajan N, Gnanasekar R, Kapil VV, Mohamed AS. Serum Chromium Levels in Type 2 Diabetic Patients and Its Association with Glycaemic Control. J Clin Diagn Res. Nov 2015; 9(11): 0C05-8.

[9] Nsonwu AC, Usoro CAO, Etukudo MH, Usoro IN. Influence of Age, Gender and Duration of Diabetes on Serum and Urine Levels of Zinc, Magnesium, Selenium and Chromium in Type 2 Diabetics in Calabar, Nigeria. Turk J Biochem.2006; 31(3): 107-14.

[10] Doddigarla Z, Parwez I, Ahmad J. Correlation of serum chromium, zinc, magnesium and SOD levels with HbA1c in type 2 diabetes: A cross sectional analysis. Diabetes Metab Syndr. Jan-Mar 2016; 10(1 Suppl 1): S126-9.

[11] Abd Elgadir A. Altoum et al. Status of some selected Trace Elements Levels and lipid profile in Ischemic heart disease patients with type 2 Diabetes Mellitus. International Journal of Pharmaceutical Research. Oct - Dec 2020; 12.

[12] Mohamed H, Sadik I, Eltom A, Osman A, Babker A. A Study of Correlation of Serum Chromium Level with Glycosylated Haemoglobin (HbA1c), Total Cholesterol and Triglycerides, among Type 2 Diabetes Patients. Open Journal of Blood Diseases. Mar 2019; 9(1): 1-8.

[13] Eva H, Akter QS, Alam MK. Relationship between Glycemic Status and Serum Chromium Level with Type 2 Diabetes Mellitus. Mymensingh Med J. Jan 2020; 29(1): 183-186. 